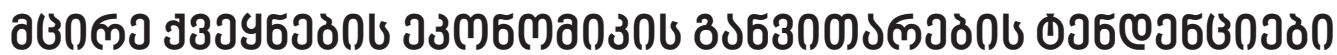

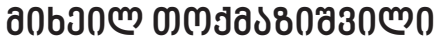

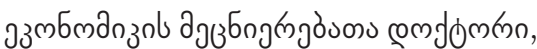

https://doi.org/10.35945/gb.2017.03.003

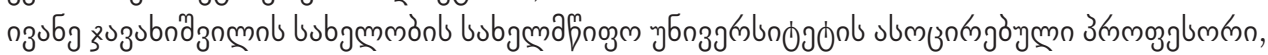

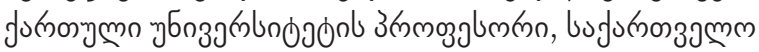

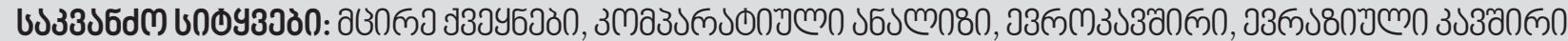

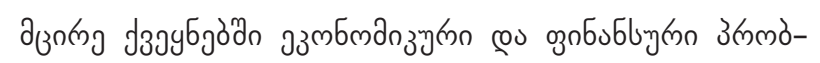

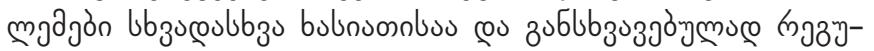

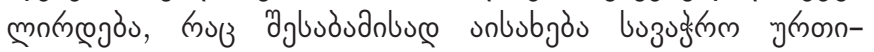

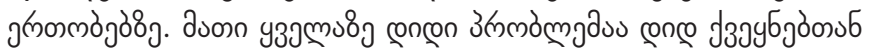

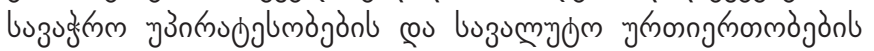

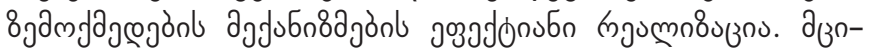

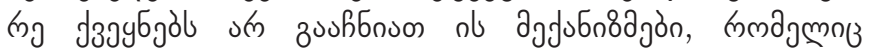

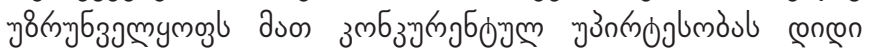

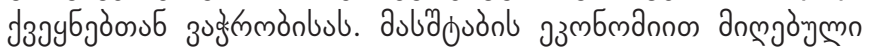

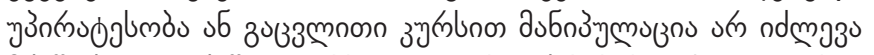

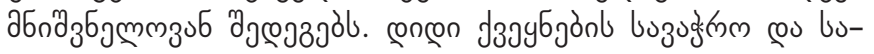
зuмmyom 8m

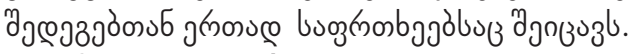

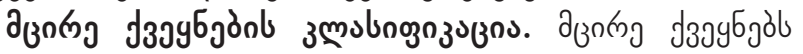

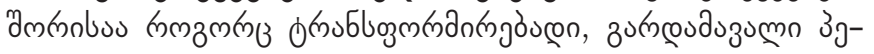

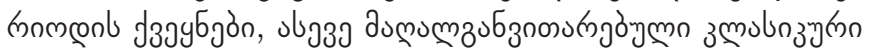

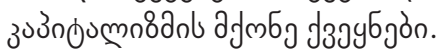

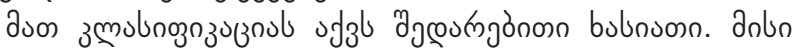

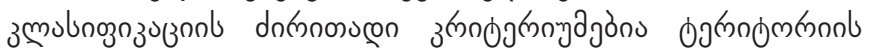

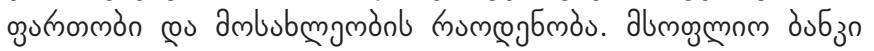

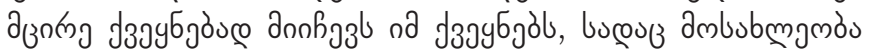

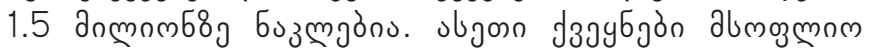

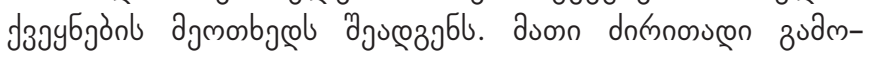

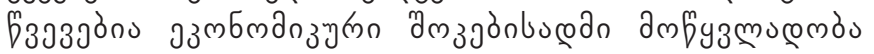

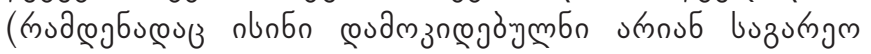

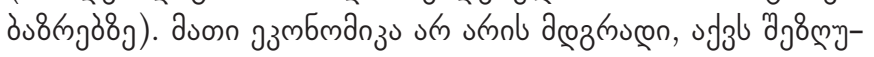

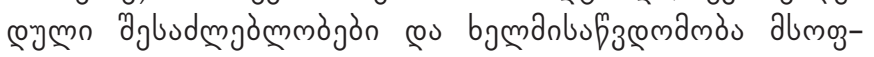

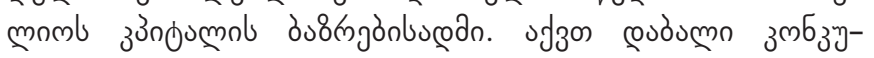

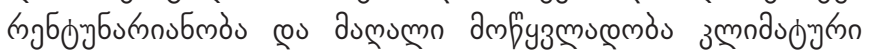

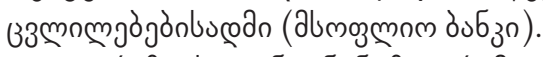

эз

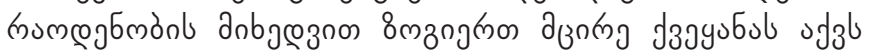

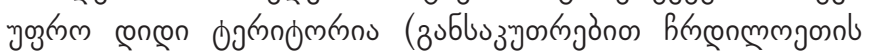

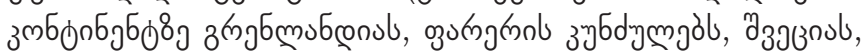

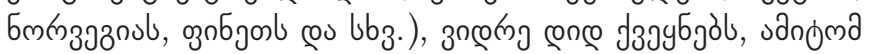

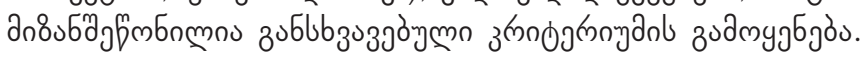

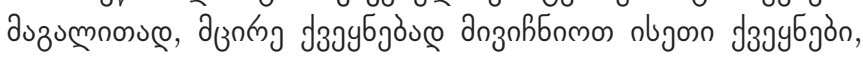

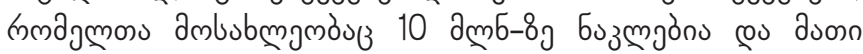

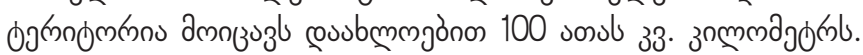

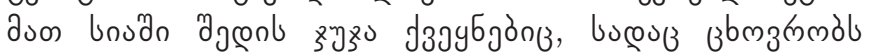

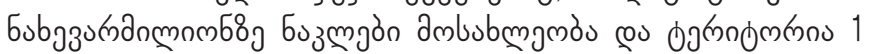

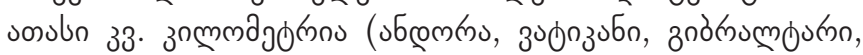

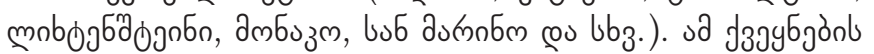

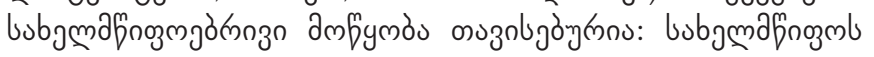

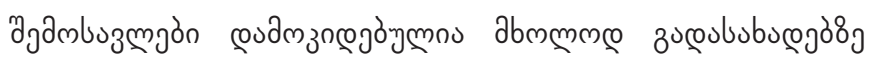

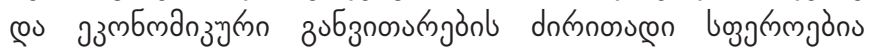

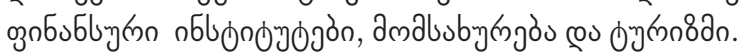

эз

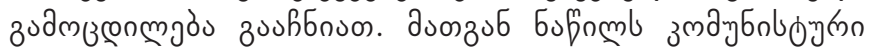

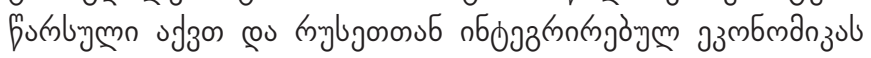
fumaucojb

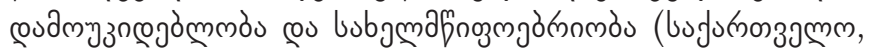

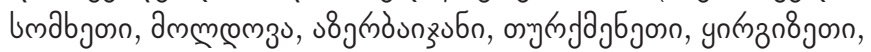

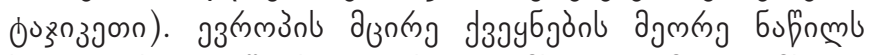

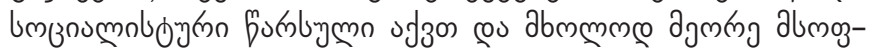

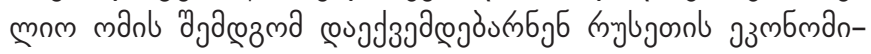

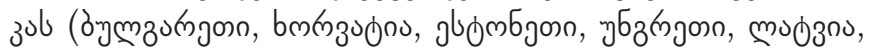

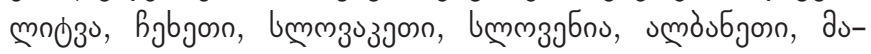

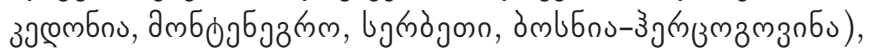

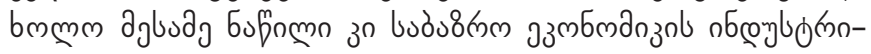

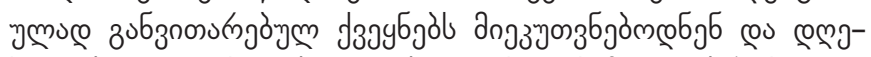

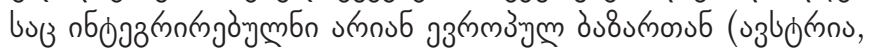

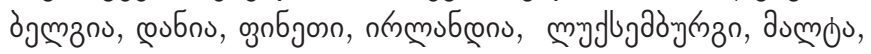

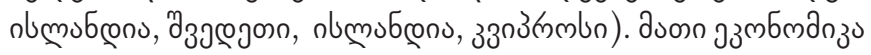

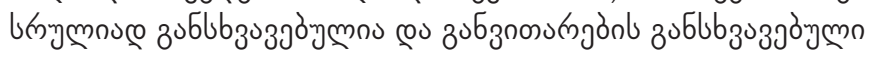
उतzmn ubulnuongón.

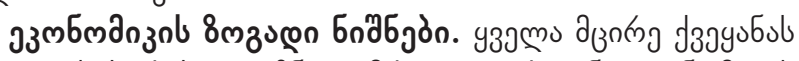

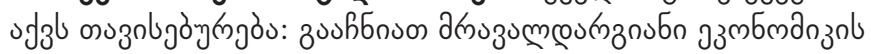

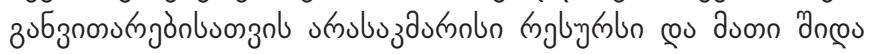

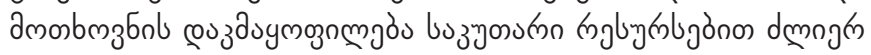

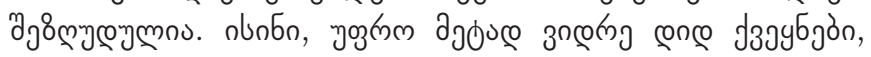

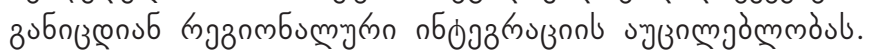

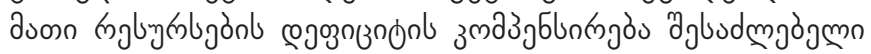

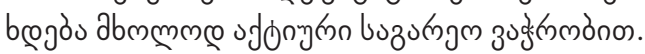

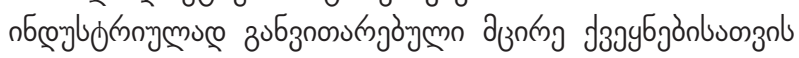

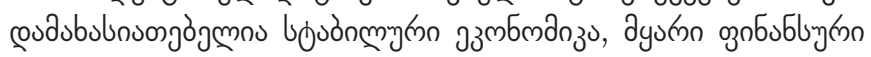

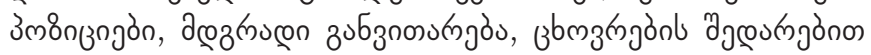
aumumo combj.

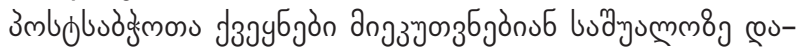

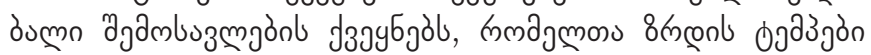

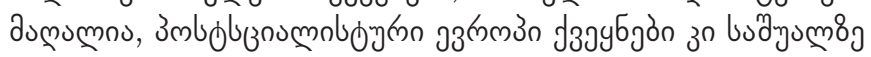

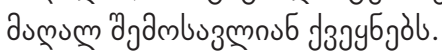

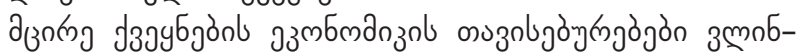

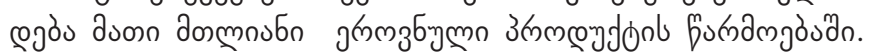

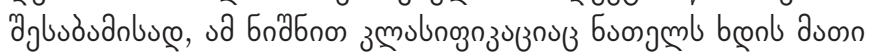
उuбznosumgònl sembgl.

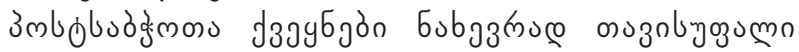

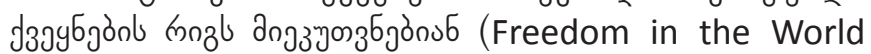




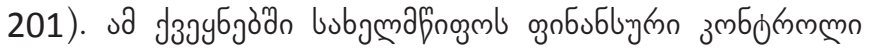

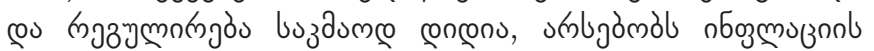

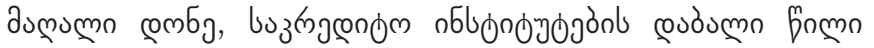

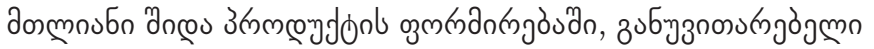

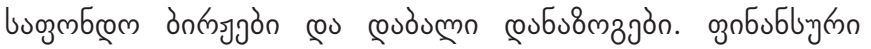

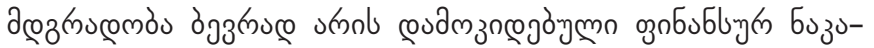
œృ

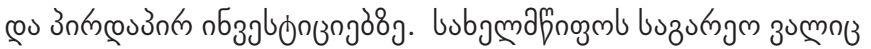

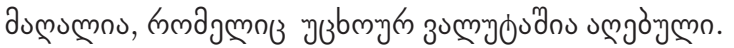

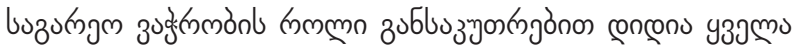

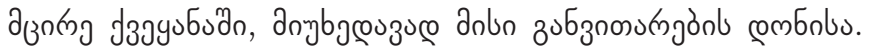

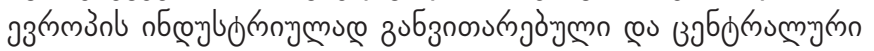

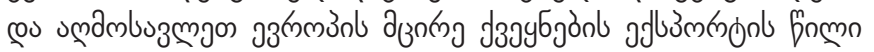

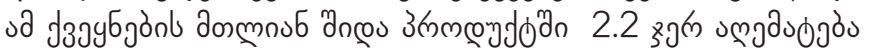

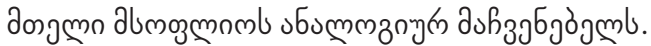

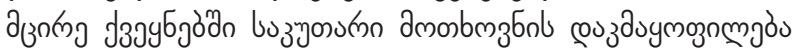

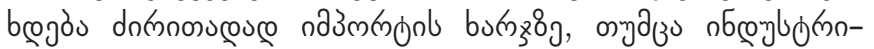

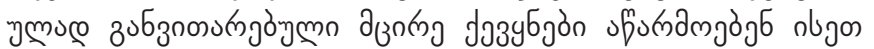

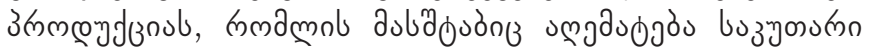

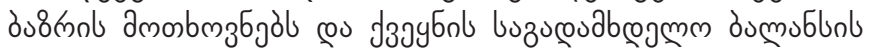

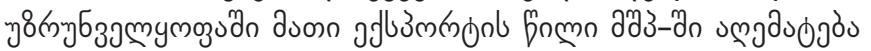

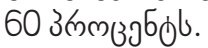

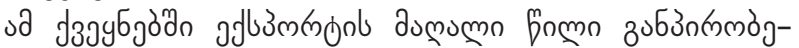

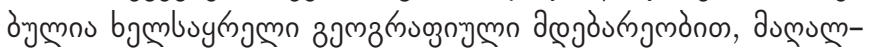

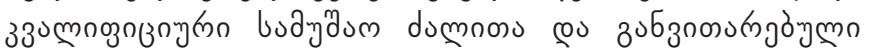

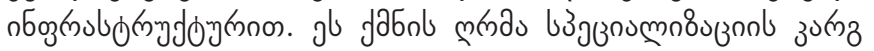

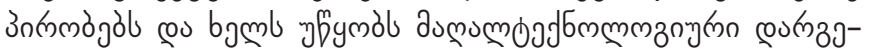

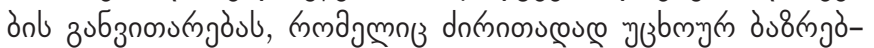

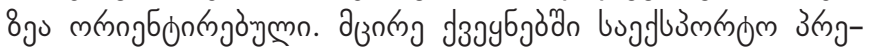

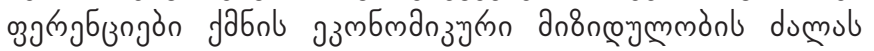

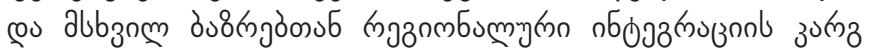
3ntrmósl.

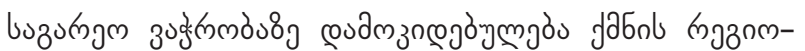

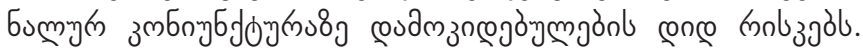

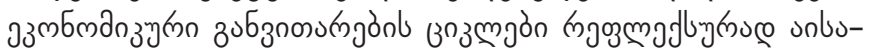

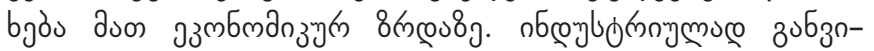

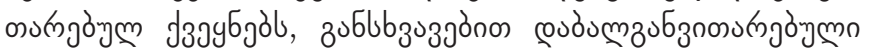

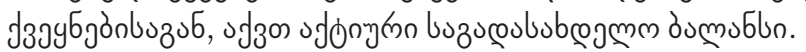

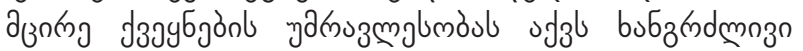

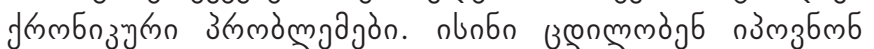

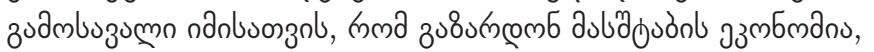

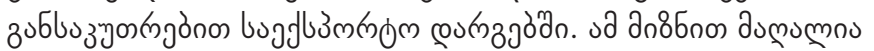

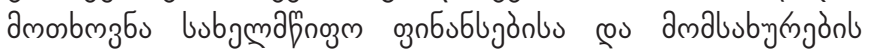

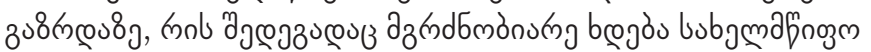

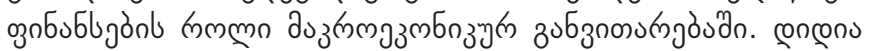

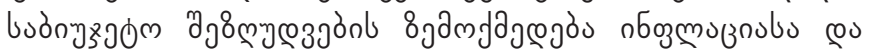

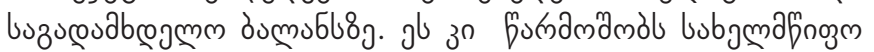

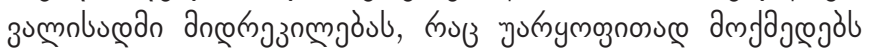

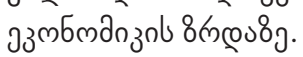

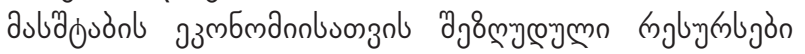

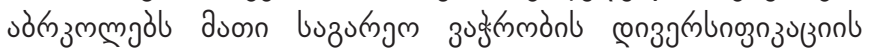

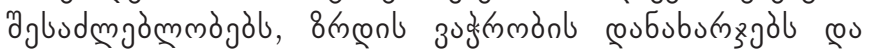

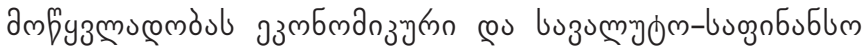

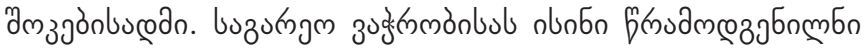

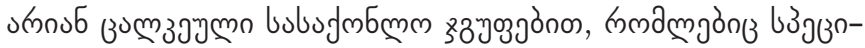

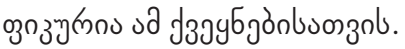

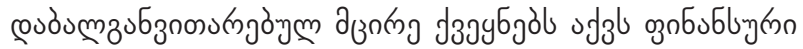

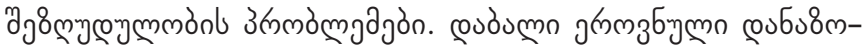

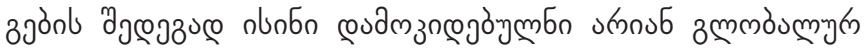

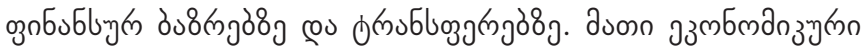

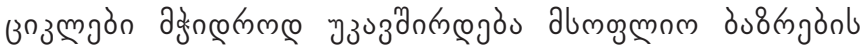

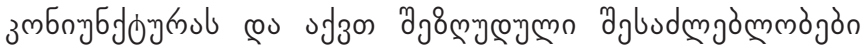

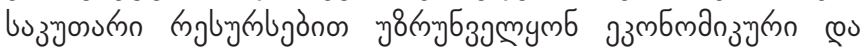

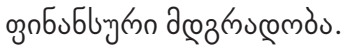

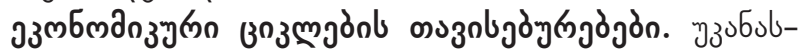

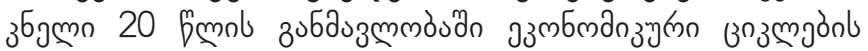

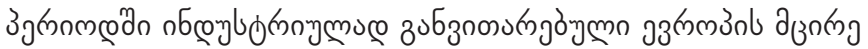

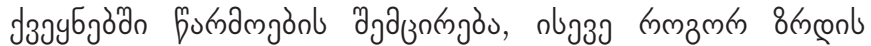

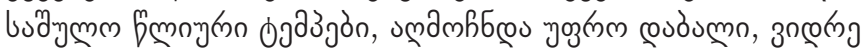

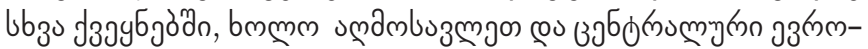

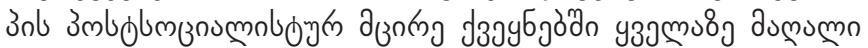

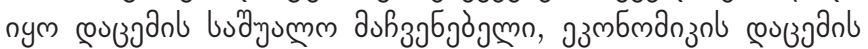

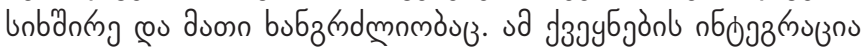

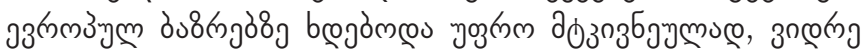

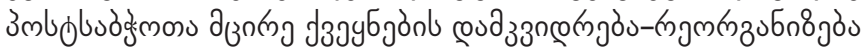

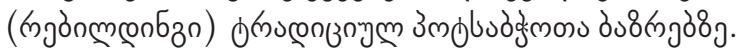

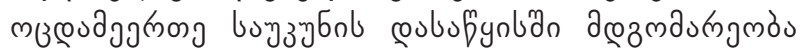

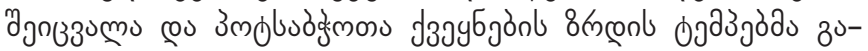

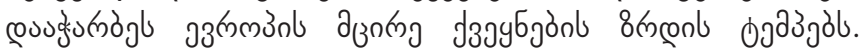

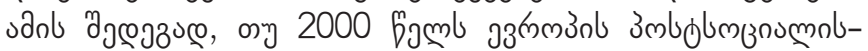

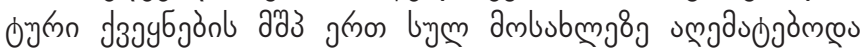

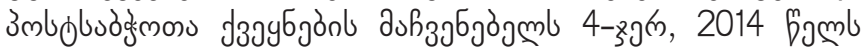

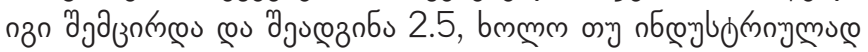

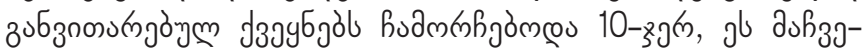

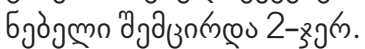

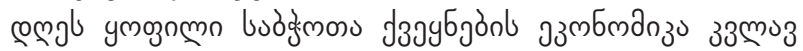

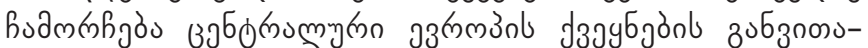

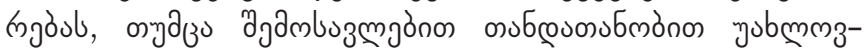

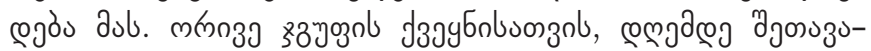

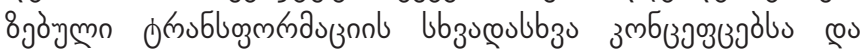

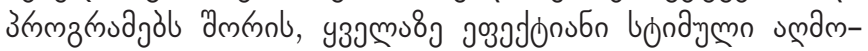

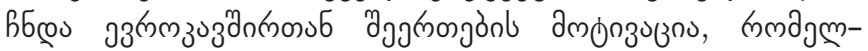

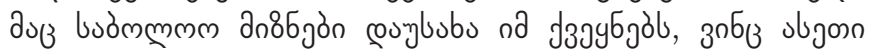

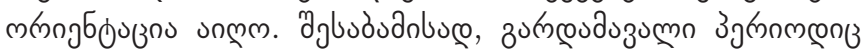

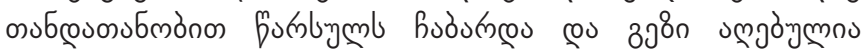

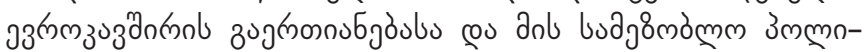

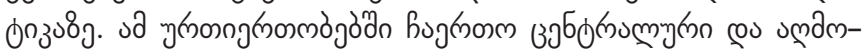

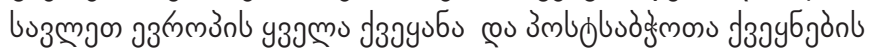

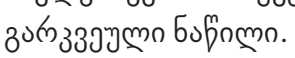

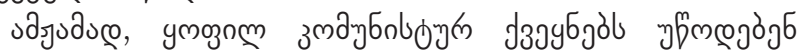

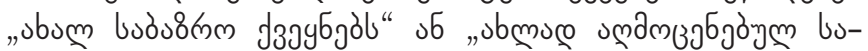

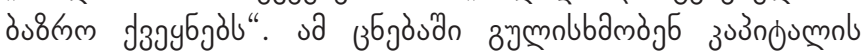

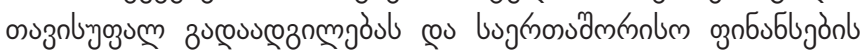

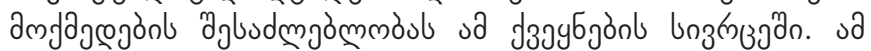




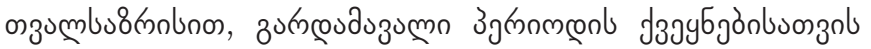

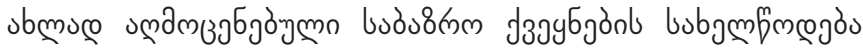
buzbjönon durnosjöymno (Silagadze A., Tokmazishvili M).

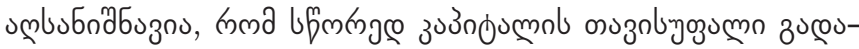

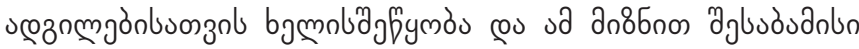

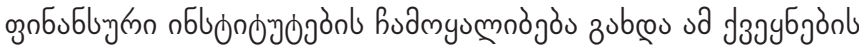

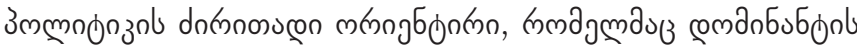

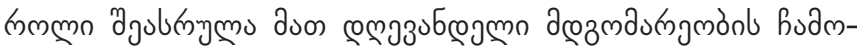

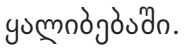

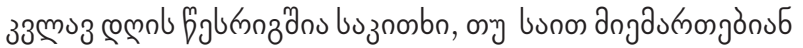

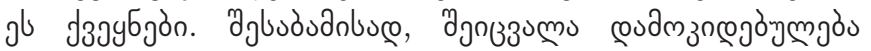

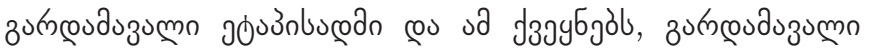

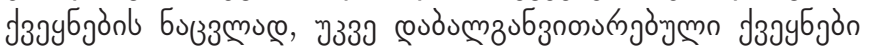

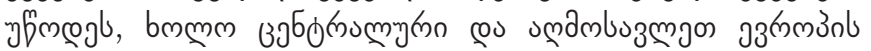

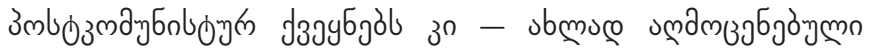

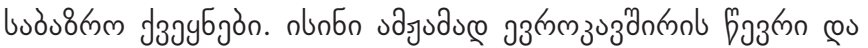

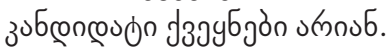

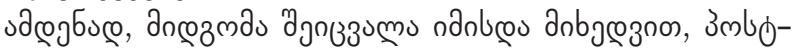

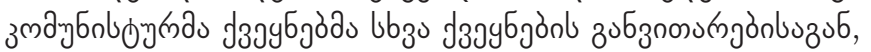

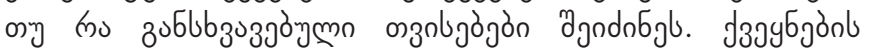

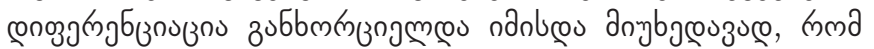

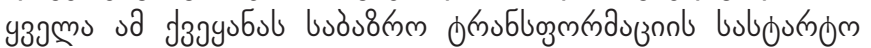

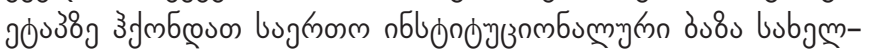

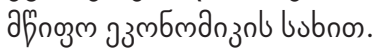

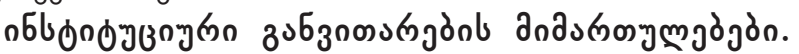

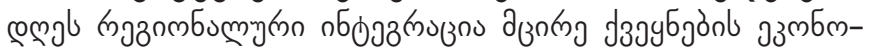

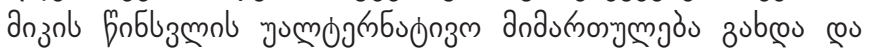
moठ̀gr

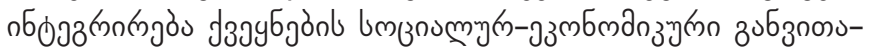

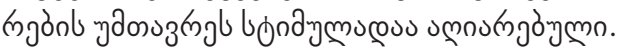

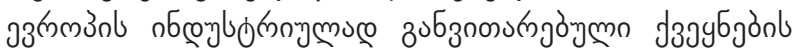

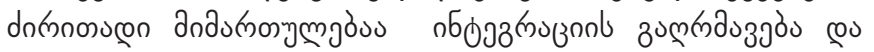

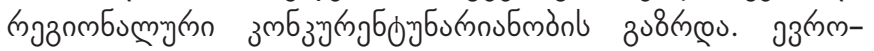

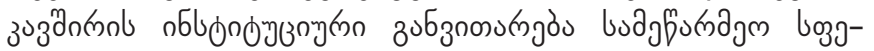

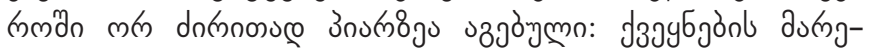

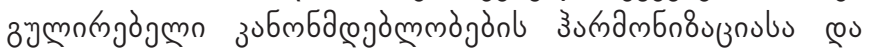

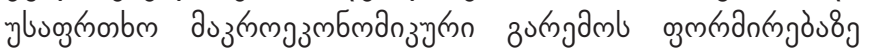

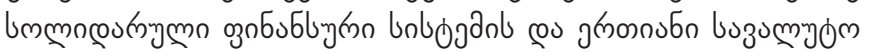

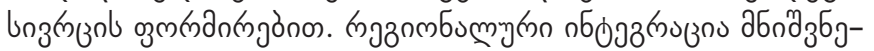

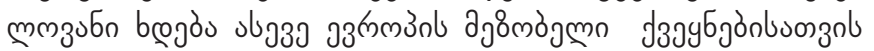

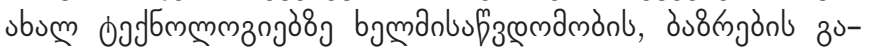

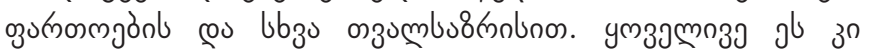

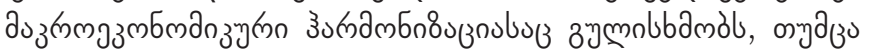

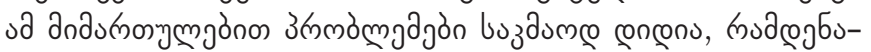

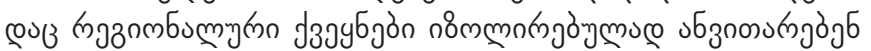

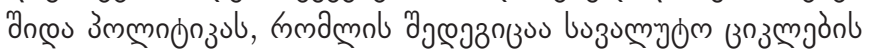

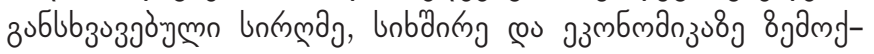

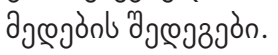

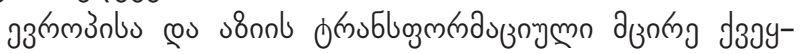

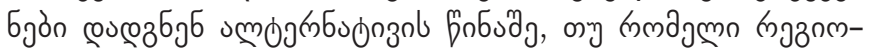

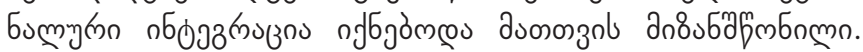

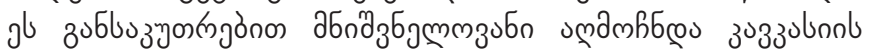

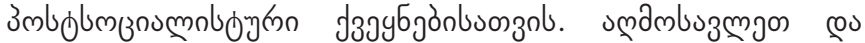

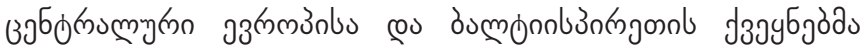

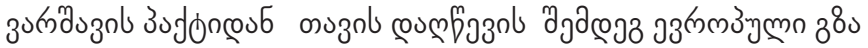

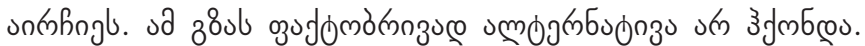

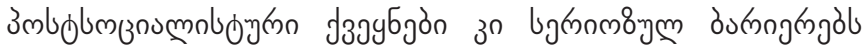

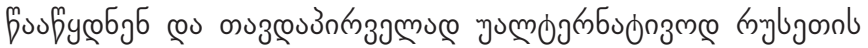

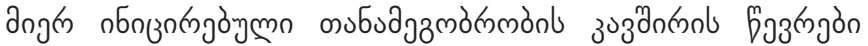

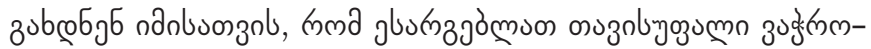

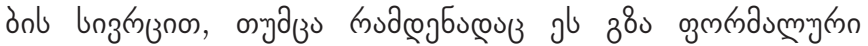

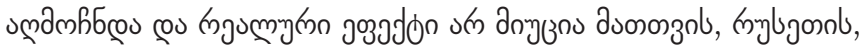

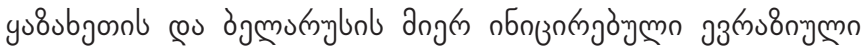

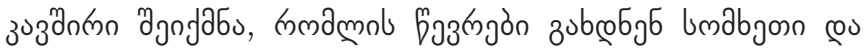

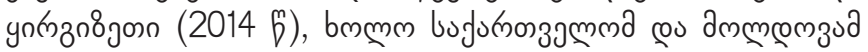

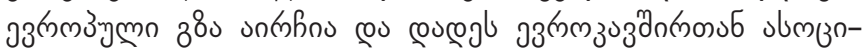

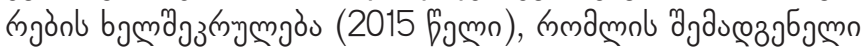

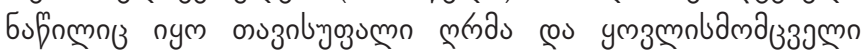

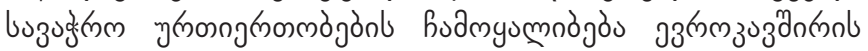

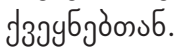

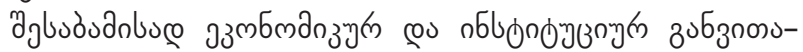

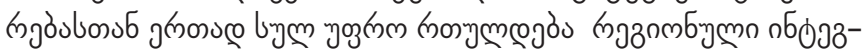

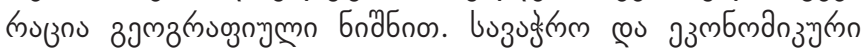

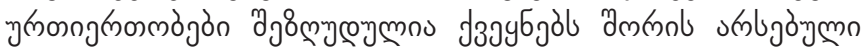

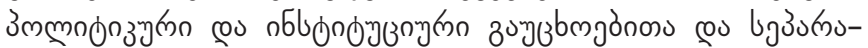

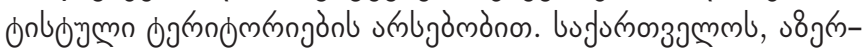

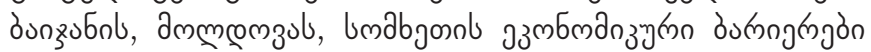

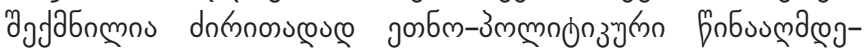

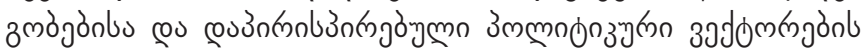

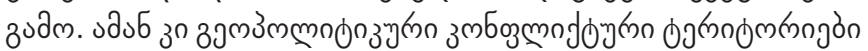
fumamdabs.

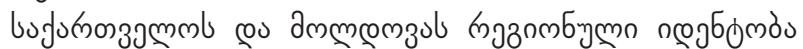

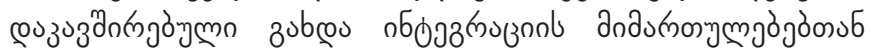

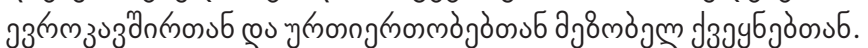

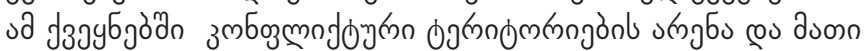

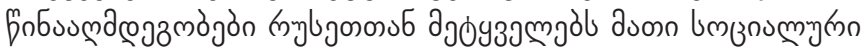

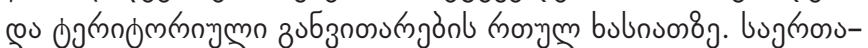

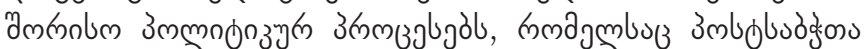

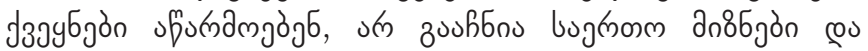

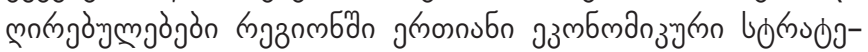

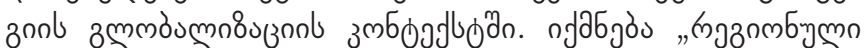

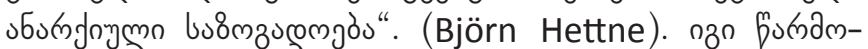

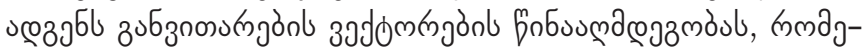

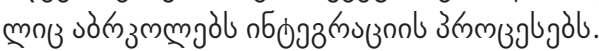

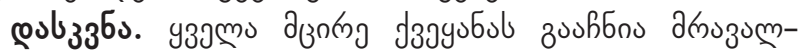

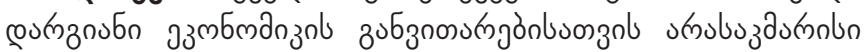

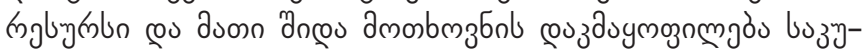

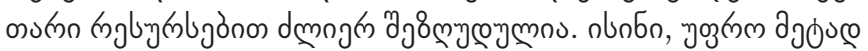

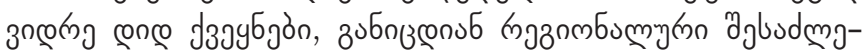

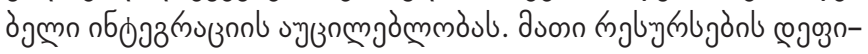

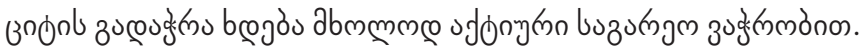

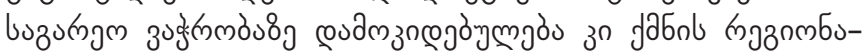

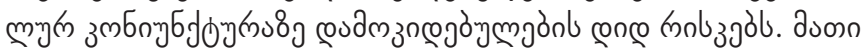

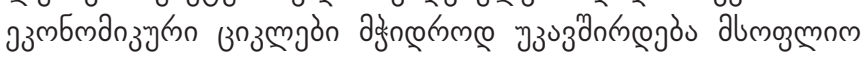




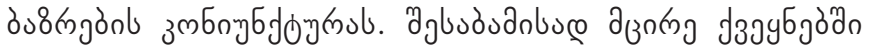

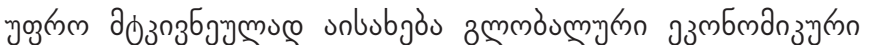

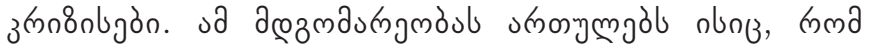

эз

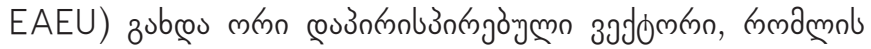

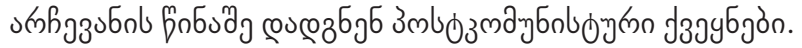

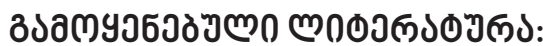

1. Björn Hettne, Globalization, the New Regionalism and East Asia, in the: Globalism and Regionalism, Ed. by Toshiro Tanaka and Takashi Inoguchi, http://archive.unu.edu/unupress/globalism.html.

2. Economic Growth and Integration of Small States to the World Economy, December 8, 2005 PRMED, http://siteresources. worldbank.org/INTDEBTDEPT/Resources

3. /468980-1206974166266/4833916-1206989877225/SmallStatesDecember2005.pdf.

4. Freedom in the World 2016. Freedom House. https://freedomhouse.org/sites/default/files/FH_FITW_Report_2016.pdf.

5. Kan Chen, Mario Crucini, (2014), Trends and Cycles in Small Open Economies: Making The Case For A General Equilibrium Approach. https://www.frbatlanta.org/-/media/Documents/news/conferences/2014/international-economics/Crucini.pdf

6. Silagadze A., Tokmazishvili M. Challenges of the Post-Communist Financial_Currency policy. Monetary, flscal and Trade Policies Series. Nova Publisher. New York.

7. The economics of small states. Big problems for little countries, Sep 10th 2014 http://www.economist.com/blogs/freeexchange/2014/09/economics-small-states

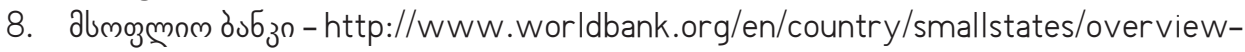




\title{
TENDENCIES OF DEVELOPMENT OF ECONOMIES OF SMALL COUNTRIES
}

\author{
MIKHEIL TOKMAZISHVILI
}

https://doi.org/10.35945/gb.2017.03.003

Doctor of Economic sciences,

associated professor of Ivane Javakhishvili Tbilisi State pofessor of Georgian University, Georgia

KEYWORDS: SMALL COUNTRIES, COMPARATIVE ANALYSIS, EU, EURASIAN UNION

\section{SUMMARY}

The economic prospects of small countries are associated with the historical and political context of their institutional development, as well as with the rules of economic regulation. These countries have different historical experience and peculiar cycles of the development, which is one of the important criteria for their classification.

All small countries have insufficient resources for the development of the economic sectors. Unlike large countries, they experience acute need for regional integration, and compensation of scarcity of the resources is possible only through the development of intensive foreign trade. Dependence on foreign trade relations creates high risks of transferring of regional problems. Accordingly, small countries are painfully impacted by the global economic crises.

Comparative analysis indicates that in the last 20 years Central and Eastern European countries are more vulnera- ble to the economic challenges, rather than the post-soviet countries. During the economic cycles production of industrialized European small countries has failed and the average annual rate of growth has declined, but reducing the economy was lower than in other countries. In the post-socialist small countries of Eastern and Central Europe declining of the average growth rate, also frequency and duration of cycles of the economy were much higher. Integration of these counties into the European markets was more painful than the process of rebuilding of post-soviet countries trade relations in the traditional historical markets.

At present the situation is complicated by the fact that the association with the EU and the Eurasian Union (DCFTA and EAEU) are two opposing vectors, which post-communist countries face to, in order to make choice for their future perspectives. 